\title{
Investing in Fundamental Research: \\ Evaluation of the Benefits that the UK Has Derived from CERN
}

\author{
Philip Amison and Neil Brown
}

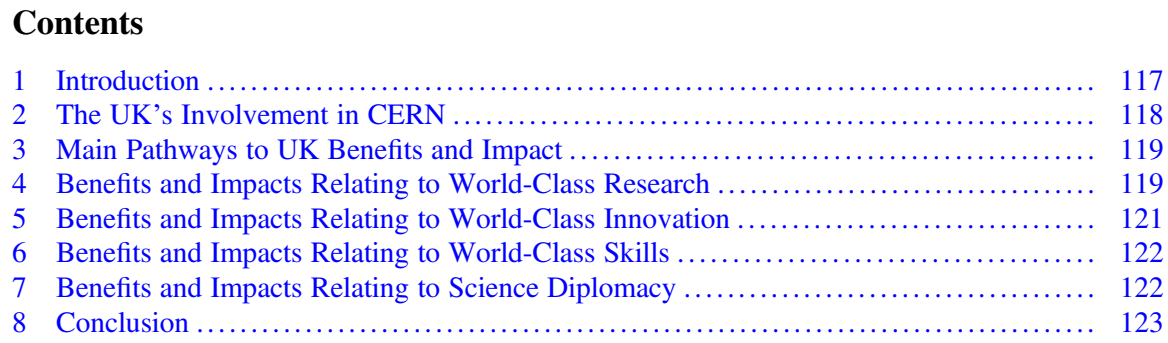

\section{Introduction}

Investing in fundamental research enables us to push the frontiers of knowledge. However, since we don't have access to unlimited resources we have to make choices about which areas to invest in. It is often easier to place a value on the costs of an investment than the benefits. Often, the benefits of funding a piece of research cannot be known in advance of making the investment, plus it may take many years for those benefits to be realised. Even in cases where we are looking back - trying to place a value on a past or ongoing investment in research-it is not always straightforward to identify, attribute and quantify the benefits and it is typically harder still to place a monetary value upon them.

Even if we think we understand the pros and cons, or the costs and benefits, of investment in research it is important to communicate and consider that understanding with a wider public, including the ultimate funders of public research, the

\section{P. Amison (ه)}

Science and Technology Facilities Council, UK Research and Innovation, Swindon, UK e-mail: philip.amison@stfc.ukri.org

N. Brown

Technopolis Group, Brussels, Belgium

(C) The Author(s) 2021 
taxpayer. Articulating the benefits from research is also important if we are to continue to inspire society more generally to engage with science and technology and encourage the next generation to study and work in this area.

To help answer questions such as these, in 2018 the Science and Technology Facilities Council (STFC) ${ }^{1}$ commissioned Technopolis $^{2}$ to undertake an evaluation of the benefits that the UK has derived from CERN. The aim was to capture, demonstrate and measure the range of scientific, economic and social impacts emerging over the past decade, considering both direct UK involvement and use, as well as any wider influences of CERN on the UK. The study drew on multiple sources of evidence, including desk research, surveys, interviews, case studies and bibliometrics, to explore the various impacts of CERN on the UK.

The following sections of this paper summarise the main findings from the study. Further information about the study can be found in the main report, which will be available soon via the UKRI web pages.

\section{The UK's Involvement in CERN}

The UK is one of CERN's founding members and has been centrally involved throughout its history. It currently contributes around $£ 150 \mathrm{~m}$ per year to the CERN budget (16\% of Member State subscriptions in 2019), which covers the building, operation and maintenance of the infrastructure, plus the governance and administration of CERN. The construction, maintenance, upgrade and operation of the experimental programme and the computing infrastructure is then mainly supported through funding from agencies of participating countries. For the UK this funding - as well as direct involvement in construction, maintenance, upgrades and operation - is mainly provided through STFC, enabling UK-based researchers to participate in the experimental programmes hosted by CERN. ${ }^{3}$ Through its subscription and broader participation the UK secures a number of benefits. These include:

- Access for UK physicists/engineers to key research infrastructure and collaboration networks;

- The opportunity for UK companies to bid for contracts, including those requiring a high intellectual and technical capacity, those that are non-technical but require high levels of expertise (e.g. financial services) and contracts for more standard supplies and services (e.g. cabling); and

\footnotetext{
${ }^{1}$ The Science and Technology Facilities Council (STFC) is part of UK Research and Innovation; www.ukri.org

${ }^{2}$ Technopolis group, United Kingdom, 3 Pavilion Buildings, Brighton BN1 1EE, T + 441,273 204,320; E neil.brown@technopolis-group.com; www.technopolis-group.com

${ }^{3}$ Other parts of UKRI and other funders also award funds relating to CERN, but on a smaller scale.
} 
- Training and work opportunities, e.g. long-term attachments for students from UK universities; visit programmes for UK schools and teachers; and apprenticeships, secondments and fellow's schemes.

It is important to note that CERN is not a user facility in the way that many other research infrastructures are (e.g. the ILL or ESRF). Rather, the UK is a partner in the co-development of the CERN facility and its programmes of work. CERN is also the UK's national laboratory for particle physics and the UK has played a key role in its strategy and development, while UK personnel have been involved in all the major experiments and discoveries. When we talk about CERN's activities and achievements, therefore, these are really the results of cross-country collaborative efforts and endeavours.

\section{Main Pathways to UK Benefits and Impact}

CERN's strands of activity and engagement are multi-faceted, with a wide range of types of benefits and impact, flowing through a series of interrelated pathways. The study defined and demonstrated 12 main areas of impact that flow to the UK, which are organised under the broad impact areas of research, innovation, skills and science diplomacy. Figure 1, below, summarises each of these impact pathways. The highlights from each of these areas are summarised in the remaining sections of this paper.

\section{Benefits and Impacts Relating to World-Class Research}

CERN research makes important advances in particle physics, including a series of landmark discoveries such as the Higgs boson-the last missing piece of the Standard Model of particle physics, hypothesised more than 40 years earlier. Other major breakthroughs made at CERN include the discovery of weak neutral currents (1970s) and electroweak (W and Z) gauge bosons (1980s), measurement of the number of lepton generations (1990s), observation of CP violation in charm quarks (2019) and the (to-date) null result showing the lack of supersymmetry. These advances support further scientific progress and offer the potential for very significant wider societal impacts in the longer term. UK scientists also build on this research to support their further progress and achievements. In the past 10 years alone, over 20,000 UK scientific papers have cited CERN articles, and this includes many of the UK's most influential physics papers (25\% of these UK papers are among the $10 \%$ most cited in their field globally).

Pooled investments by Member States have enabled facilities that would be beyond the reach of any country alone, providing access and opportunities at world leading facilities for UK researchers at a fraction of the overall costs. This 


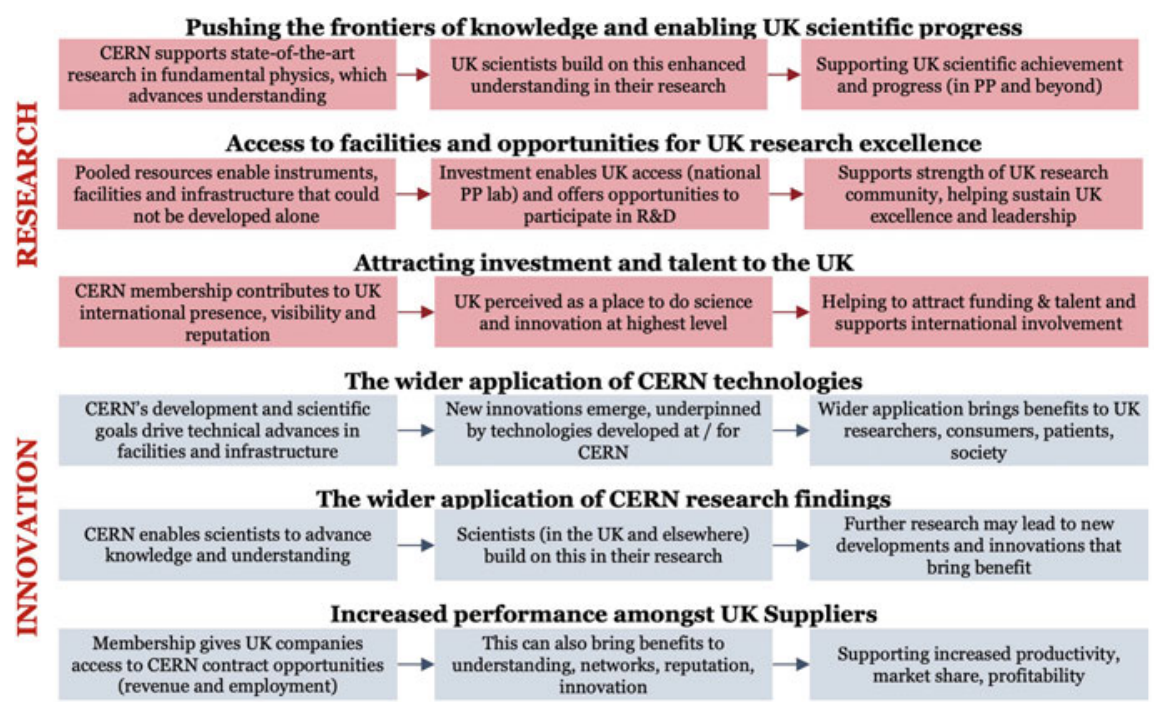

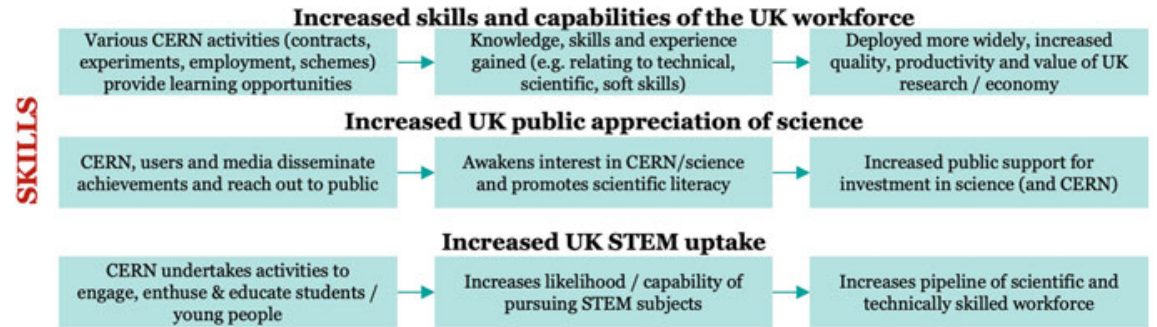

\begin{tabular}{|c|c|c|c|}
\hline \multicolumn{4}{|c|}{ The UK's influence in the international S\&T landscape } \\
\hline $\begin{array}{l}\text { Membership allows UK involvement } \\
\text { / influence in CERN governance and } \\
\text { wider agenda setting fora }\end{array}$ & $\rightarrow$ & $\begin{array}{l}\text { Offering opportunity to influence } \\
\text { decision making (funding \& } \\
\text { priorities) within and beyond CERN }\end{array}$ & $\begin{array}{l}\text { Increased UK-relevance and 'fit' of } \\
\text { opportunities, activities and } \\
\text { outcomes }\end{array}$ \\
\hline \multicolumn{4}{|c|}{ The UK's image as a 'great science and innovation nation' } \\
\hline $\begin{array}{l}\text { CERN is highly visible \& well } \\
\text { regarded so UK's role within CERN } \\
\text { provides international visibility }\end{array}$ & & $\begin{array}{l}\text { Nurture perceptions of the UK as a } \\
\text { 'great science and innovation nation' } \\
\text { (open, experienced, committed) }\end{array}$ & $\begin{array}{c}\text { Foreign governments, business \& } \\
\text { public more interested in /inclined to } \\
\text { engage with the UK }\end{array}$ \\
\hline \multicolumn{4}{|c|}{ Improved diplomatic relations and engagement } \\
\hline $\begin{array}{l}\text { CERN provides a platform ( \& 'neutral } \\
\text { space') for international } \\
\text { collaboration \& exchange }\end{array}$ & & $\begin{array}{l}\text { Builds relationships, trust and } \\
\text { processes of engagement between } \\
\text { countries }\end{array}$ & $\begin{array}{l}\text { UK benefits from interaction / relations, } \\
\text { and indirectly from wider relations (re: } \\
\text { global challenges, peace/security) }\end{array}$ \\
\hline
\end{tabular}

Fig. 1 Main pathways to UK benefits and impacts from CERN. Source: Evaluation of the Benefits that the UK has derived from CERN, Technopolis Group, 2019

includes access to unique technologies and capabilities, international collaborations and networks, frontier science and experiments, the latest theories and developments, new methods, training and learning opportunities. The UK science and engineering community is taking up such opportunities on a significant scale, with over 1000 researchers from $30 \mathrm{UK}$ organisations currently using CERN (third highest amongst Member States). 
CERN opportunities support the strength of the UK research community, helping to sustain the UK as a world leading research nation. UK personnel have been involved in all of the major experiments and discoveries at CERN, with many UK researchers holding key positions. CERN-based publications have also significantly pulled the UK's citation metrics upwards, demonstrating the high-quality research enabled. In addition, CERN contributes to the UK's international presence, visibility and reputation, which plays out through the attraction of funding, talent and other forms of recognition for the UK.

\section{Benefits and Impacts Relating to World-Class Innovation}

CERN's scientific breakthroughs have required major advances in technologies, which have then found wider application, across research and industry. In several notable cases (e.g. the World Wide Web, detectors for PET scanners, touchscreens, GRID computing), CERN has provided the platform for a major new technology that has come into general use and had a transformative effect, bringing economic and societal benefits to the UK and the rest of the world. Other examples of innovations emerging from CERN include the HTTP protocol, next-generation (hadron beam) radiotherapy, radiation-hardened robotics for nuclear decommissioning, fibre optic sensors to help manage water shortages and various software tools and advances in machine learning, pattern recognition and big data analyses (amongst many others). Forthcoming upgrades to the facility will require further technological innovation, which in time will no doubt also find wider uptake and application beyond CERN and particle physics.

CERN membership also gives UK companies access to a steady stream of contract opportunities, with around 500 UK firms having sold goods and services to CERN in the past decade, bringing in an additional $£ 183 \mathrm{~m}$ in revenue and supporting employment (all figures in 2018 prices, unless stated). In addition, at least $£ 33 \mathrm{~m}$ was awarded to UK firms for CERN experiments (organised by collaborating countries) and by the CERN Pension Fund. These contracts have been won by a wide range of UK firms, from small precision engineering companies, through to global technology firms and pension fund managers.

These UK suppliers also realise wider benefits, beyond the value of the contracts themselves, for instance through the development of innovative technologies or access to new market opportunities. CERN contracts also bestow a degree of prestige on suppliers that is not easily replicated elsewhere, which aids new sales. Half of UK suppliers reported that past CERN contracts had resulted in an increase in other sales income, and the study estimates that a further $£ 1$ billion in turnover and $£ 110 \mathrm{~m}$ in profit has been supported amongst UK suppliers in the past decade, on top of the direct income received through contracts. 


\section{Benefits and Impacts Relating to World-Class Skills}

There is significant uptake of CERN training opportunities in the UK. In the past decade, around 1000 individuals have participated across the various formal schemes that CERN offers, receiving (free) training worth more than $£ 4.9 \mathrm{~m}$. Even more acquire skills and knowledge 'on the job', including each year around 1000 researchers, 300 CERN staff, 40 fellows and hundreds of individuals at UK suppliers. Through these interactions, the UK workforce gains knowledge and skills across a variety of areas (technical, scientific, digital, problem solving) through an experience that is considered near-unique. Young UK researchers who have engaged with CERN are estimated to earn $12 \%$ more across their careers as a result (with an extra $£ 489 \mathrm{~m}$ in additional wages realised in the past 10 years alone).

The knowledge and skills gained via CERN are also deployed more widely in the UK economy. Students, researchers and staff move to various roles (analysts, scientists, engineers, developers, management) across a variety of sectors (IT and software, engineering, manufacturing, financial services, health) in the public, private and third sectors. Their capabilities are in great demand, with shortages of STEM skills in general costing UK firms $£ 1.5$ billion a year in recruitment, temporary staff and additional training. CERN, researchers and the media also disseminate and reach out to the wider UK public. Each year (on average) from the UK: 12,000 school students and other members of the public visit CERN in person; 220,000 visit CERN's website; and 40,000 interact with its social media. There are also 2000 mentions of CERN in the UK media each year, plus various TV/Radio broadcasts. CERN helps to increase the UK public's appreciation of science, awakening interest in CERN, the science that it supports and the benefits of this work. This helps promote scientific literacy and in the development of a culture valuing science. The results of a separate study (Florio, 2018) suggest that the UK public would be willing to pay (through taxation) around $£ 1.2$ billion for CERN over a decade-more than the UK's actual contributions.

CERN also undertakes activities specifically aimed at engaging, enthusing and educating young people. The UK has the most teachers attending the CERN National Teacher Programme (over 1000 in the past decade), who go on to teach an estimated 175,000 school students with context from CERN within 3 months of the visit. As mentioned above, thousands of UK pupils also visit CERN each year, increasing the likelihood and capability of young people pursuing STEM subjects at A-level and university.

\section{Benefits and Impacts Relating to Science Diplomacy}

The UK is actively involved at all levels of CERN governance, providing UK ministries, funding agencies, and the wider UK science base with an important platform for international engagement, leadership and agenda-setting. CERN also 
provides a platform for the UK to engage more widely in global initiatives and international networks. CERN is highly visible and well regarded internationally, which spills over to favourable perceptions of its members and greater engagement (in science, technology and beyond).

CERN has been instrumental in science diplomacy, with a constitutionallydefined policy of openness and a commitment to provide a neutral space for global collaboration, the importance of which was recognised when CERN was granted observer status at the UN General Assembly. CERN also actively seeks to establish links with and promote research by countries across the globe, facilitating cooperation among the scientists and policy makers of many countries that are experiencing strained relations at the political level. CERN is an example of science for peace that has inspired several other major cooperative initiatives. For example, the SESAME synchrotron light source showcases the critical role that CERN has played in fostering cooperation across political, religious and cultural divides in the Middle East.

CERN has also nurtured the global physics community through the development of young researchers (particularly in less well-endowed scientific communities) and via collaborations. UK scientists have been at the centre of this outreach work that has resulted in many countries - from Argentina to South Africa-engaging with CERN experiments and investing in their own national researchers and facilities, with improved local capabilities and wider international research collaborations resulting.

\section{Conclusion}

CERN addresses fundamental questions about the Universe at a facility of unprecedented scale. It is the world's largest particle physics laboratory, making available complex, purpose-built particle accelerators and detectors, as well as computing technology, for its global research community. Its strands of activity and engagement are multi-faceted-from fundamental research to discover new particles and forces, through to school visits that inspire tomorrow's scientists—and this study has provided the most thorough attempt yet to capture and measure the full range of benefits that flow from CERN, covering impacts relating to research, innovation, skills and science diplomacy.

Monetising such impacts is challenging, however, as the laboratory's contributions to society unfold over decades, with its advances in knowledge and technology helping to underpin manifold social and economic benefits that occur in many different places and in a somewhat unpredictable fashion. Moreover, the realisation of these benefits invariably depends, in part at least, on many accompanying developments and wider events. In addition to these CERN-derived knowledge spillovers, there are also undeniable benefits of being a member and 'sitting at the table' where decisions are being made today about the future of particle physics 20-30 years hence. 
Evaluators are gradually developing tools to overcome the particular methodological challenges of tracing and measuring the impact of public investment in Big Science, and Technopolis' study for STFC used a range of different state-of-the-art approaches to capture and monetise some of the most important impacts attributable to CERN, demonstrating over $£ 1$ billion-worth of benefits for the UK in the period 2009-2018. This is a lower bound estimate of overall impact and doesn't, for instance, include wider technology spillovers (e.g. the World Wide Web), which if monetised would be substantial. Even so, it already largely justifies the cost of UK investment in the facility, and provides a good first indication of the scale of potential benefits that flow from our investment in such large scale research infrastructures.

Open Access This chapter is licensed under the terms of the Creative Commons Attribution 4.0 International License (http://creativecommons.org/licenses/by/4.0/), which permits use, sharing, adaptation, distribution and reproduction in any medium or format, as long as you give appropriate credit to the original author(s) and the source, provide a link to the Creative Commons licence and indicate if changes were made.

The images or other third party material in this chapter are included in the chapter's Creative Commons licence, unless indicated otherwise in a credit line to the material. If material is not included in the chapter's Creative Commons licence and your intended use is not permitted by statutory regulation or exceeds the permitted use, you will need to obtain permission directly from the copyright holder. 